\title{
Minimal skew energy of oriented unicyclic graphs with fixed diameter
}

\author{
Xiang-Hao Yang*, Shi-Cai Gong and Guang-Hui Xu
}

"Correspondence: xhao@shdv.com School of Science, Zhejiang A\&F

University, Hangzhou, 311300, China

\begin{abstract}
Let $S\left(G^{\sigma}\right)$ be the skew adjacency matrix of the oriented graph $G^{\sigma}$, which is obtained from a simple undirected graph $G$ by assigning an orientation $\sigma$ to each of its edges. The skew energy of an oriented graph $G^{\sigma}$ is defined as the sum of all singular values of $S\left(G^{\sigma}\right)$. For any positive integer $d$ with $3 \leq d \leq n-2$, we in this paper, determine the graph with minimal skew energy among all oriented unicyclic graphs on $n$ vertices with fixed diameter $d$.
\end{abstract}

MSC: $05 C 50 ; 15 \mathrm{~A} 18$

Keywords: oriented graph; unicyclic graph; skew energy; diameter

\section{Introduction}

Research on the energy of a matrix in terms of a related graph can be traced back to 1970s [1] when Gutman investigated the energy with respect to the adjacency matrix of an (undirected) graph, which has a still older chemical origin; see [2]. Then much attention has been devoted to the energy of the adjacency matrix of a graph; see [1,3-8], and the references cited therein. Recently, in analogy to the energy of the adjacency matrix, a few other versions of graph energy were introduced in the mathematical literature, such as Laplacian energy [9], signless Laplacian energy [10] and skew energy [11].

Let $G$ be a simple undirected graph with an orientation $\sigma$, which assigns to each edge a direction so that $G^{\sigma}$ becomes an oriented graph. Then $G$ is usually called the underlying graph of $G^{\sigma}$. The skew-adjacency matrix associated to the oriented graph $G^{\sigma}$ with vertex set $\{1,2, \ldots, n\}$ is defined as the $n \times n$ matrix $S\left(G^{\sigma}\right)=\left[s_{i j}\right]$ whose $(i, j)$ th entry satisfies:

$$
s_{i j}= \begin{cases}1 & \text { if there is an } \operatorname{arc} \text { with head } i \text { and tail } j ; \\ -1 & \text { if there is an } \operatorname{arc} \text { with head } j \text { and tail } i \\ 0 & \text { otherwise. }\end{cases}
$$

Then $S\left(G^{\sigma}\right)$ is a skew-symmetric matrix, and thus the eigenvalues of $S\left(G^{\sigma}\right)$ are all purely imaginary numbers.

In [11], Adiga et al. introduced the concept the skew energy of an oriented graph $G^{\sigma}$, denoted by $\mathscr{E}\left(G^{\sigma}\right)$, which is defined as

$$
\mathcal{E}_{s}\left(G^{\sigma}\right)=\sum_{i=1}^{n}\left|\lambda_{i}\right|
$$

o 2013 Yang et al.; licensee Springer. This is an Open Access article distributed under the terms of the Creative Commons Attribution License (http://creativecommons.org/licenses/by/2.0), which permits unrestricted use, distribution, and reproduction in any medium, provided the original work is properly cited. 
where $\left\{\lambda_{1}, \lambda_{2}, \ldots, \lambda_{n}\right\}$ are all eigenvalues of the skew adjacency matrix $S\left(G^{\sigma}\right)$. Adiga et al. [11] showed that the skew energy of an oriented tree is independent of its orientation, which is equal to the energy of its underlying tree. Moreover, Adiga et al. [11] investigated the skew energies of oriented cycles by computing. Then Hou et al. [12] determined the oriented unicyclic graphs with minimal and maximal skew energy, respectively; Gong et al. determined all oriented graphs with minimal skew energy among all connected oriented graphs on $n$ vertices with $m(n-1 \leq m \leq 2(n-2))$ edges [13], and all 3-regular connected oriented graphs with optimal skew energies [14].

In this paper, we continue to investigate the skew energy of oriented unicyclic graphs. Below, we focus on the graphs with order at least 5 , since the skew energy of an oriented graph with a small order can be calculated directly by mathematical software such as Matlab 7.0. As we know that $2 \leq d\left(G^{\sigma}\right) \leq n-2$ for any oriented unicyclic graph $G^{\sigma}$ with order $n(n \geq 6)$. If $d\left(G^{\sigma}\right)=2$, then, up to isomorphism, $G^{\sigma}$ must be the graph obtained from the oriented star $S_{n}$ by adding one arc between arbitrary two pendent vertices of it. Therefore, we in the following always assume that $3 \leq d \leq$ $n-2$.

The rest of this paper is organized as follows: In Section 2, we give some notation and preliminary results, which will be used in the following discussion. The graph with minimal skew energy among all oriented unicyclic graphs on $n(\geq 6)$ vertices with diameter $d$ $(3 \leq d \leq n-2)$ will be determined in Section 3.

\section{Preliminary results}

Let $G=(V(G), E(G))$ be a simple graph. Denote by $G-e$ the graph obtained from $G$ by deleting the edge $e$ and by $G-v$ the graph obtained from $G$ by deleting the vertex $v$ together with all edges incident to it and by $d(G)$ the diameter of $G$, which is defined as the greatest distance between any two vertices in $G$. The union of the graphs $G_{1}=\left(V\left(G_{1}\right), E\left(G_{1}\right)\right)$ and $G_{2}=\left(V\left(G_{2}\right), E\left(G_{2}\right)\right)$, denoted by $G_{1} \cup G_{2}$, is the graph with vertex-set $V\left(G_{1}\right) \cup V\left(G_{2}\right)$ and edge-set $E\left(G_{1}\right) \cup E\left(G_{2}\right)$. An $r$-matching of $G$ is a subset with $r$ edges such that every vertex of $V(G)$ is incident with at most one edge in it. Denote by $m(G, r)$ the number of $r$-matchings contained in G. We refer to Cvetković et al. [15] for more terminology and notation not defined here.

For convenience, in terms of defining subgraph, matchings, degree, diameter, etc., of an oriented graph, we focus only on its underlying graph. Moreover, we will briefly use the notations $C_{n}, S_{n}$ and $P_{n}$ to denote the oriented cycle, the oriented star and the oriented path on $n$ vertices, respectively, if no conflict exists there.

An even cycle $C$ is called oddly oriented if for either choice of direction of traversing around $C$, the number of edges of $C$ directed in the direction of traversal is odd. Since $C$ is even, this is clearly independent of the initial choice of direction of traversal. Otherwise, such an even cycle $C$ is called evenly oriented. (We here do not involve the parity of the cycle with length odd, the reason is it dependents on the initial choice of direction of traversal.)

An oriented graph $H$ is called a 'basic oriented graph' if each component of $H$ is $K_{2}$ or a cycle with length is even. 
Denote by $\phi\left(G^{\sigma}, \lambda\right)$ the skew characteristic polynomial of the oriented graph $G^{\sigma}$, which is defined as

$$
\phi\left(G^{\sigma}, \lambda\right)=\operatorname{det}\left(\lambda I_{n}-S\left(G^{\sigma}\right)\right)=\sum_{i=0}^{n}(-1)^{i} a_{i}\left(G^{\sigma}\right) \lambda^{n-i}
$$

where $I_{n}$ denotes the identity matrix of order $n$.

The following result is a cornerstone of our discussion below, which gives an interpretation of all coefficients of the skew characteristic polynomial of an oriented graph in terms of its basic oriented subgraphs.

Proposition 2.1 ([16, Corollary 2.4], [17, Theorem 2.3]) Let $G^{\sigma}$ be an oriented graph on $n$ vertices with skew characteristic polynomial

$$
\phi\left(G^{\sigma}, \lambda\right)=\sum_{i=0}^{n}(-1)^{i} a_{i} \lambda^{n-i}=\lambda^{n}-a_{1} \lambda^{n-1}+a_{2} \lambda^{n-2}+\cdots+(-1)^{n-1} a_{n-1} \lambda+(-1)^{n} a_{n} .
$$

Then $a_{i}=0$ if $i$ is odd; and

$$
a_{i}=\sum_{\mathscr{H}}(-1)^{c^{+}(\mathscr{H})} 2^{c(\mathcal{H})} \quad \text { if } i \text { is even, }
$$

where the summation is over all basic oriented graphs $\mathscr{H}$, of $\mathrm{G}^{\sigma}$, having $i$ vertices, and $c^{+}(\mathscr{H})$ and $c(\mathscr{H})$ are respectively the number of evenly oriented even cycles and even cycles contained in $\mathscr{H}$.

As an analogy to the Coulson integral formula for the energy of an undirected graph with respect to its adjacency matrix, Hou et al. [12] deduce an integral formula for the skew energy of an oriented graph in terms of the coefficients of its skew characteristic polynomial.

Lemma 2.2 [12, Theorem 2.6] Let $G^{\sigma}$ be an oriented graph with order $n$. Then

$$
\mathcal{E}_{s}\left(G^{\sigma}\right)=\frac{1}{\pi} \int_{-\infty}^{+\infty} \lambda^{-2} \ln \psi\left(G^{\sigma}, \lambda\right) d \lambda
$$

where

$$
\psi\left(G^{\sigma}, \lambda\right)=\sum_{i=0}^{\left\lfloor\frac{n}{2}\right\rfloor} a_{2 i}\left(G^{\sigma}\right) \lambda^{2 i}
$$

and $a_{2 i}\left(G^{\sigma}\right)$ denotes the coefficient of $\lambda^{n-2 i}$ in $\phi\left(G^{\sigma}, \lambda\right)$.

From Lemma 2.2, for an oriented graph $G^{\sigma}$, the skew energy $\mathcal{E}_{s}\left(G^{\sigma}\right)$ is a strictly monotonically increasing function of the coefficients $a_{2 k}\left(G^{\sigma}\right)\left(k=0,1, \ldots,\left\lfloor\frac{n}{2}\right\rfloor\right)$. Thus, similar to comparing the energies of two undirected graphs with respect to their adjacency matrices, we define the quasi-ordering relation ' $\preceq$ ' for oriented graphs as follows.

Let $G_{1}^{\sigma_{1}}$ and $G_{2}^{\sigma_{2}}$ be two oriented graphs of order $n$. $\left(G_{1}\right.$ is not necessary different from $G_{2}$.) If $a_{2 i}\left(G_{1}^{\sigma_{1}}\right) \leq a_{2 i}\left(G_{2}^{\sigma_{2}}\right)$ for all $i$ with $0 \leq i \leq\left\lfloor\frac{n}{2}\right\rfloor$, then we write that $G_{1}^{\sigma_{1}} \preceq G_{2}^{\sigma_{2}}$. 
Furthermore, if $G_{1}^{\sigma_{1}} \preceq G_{2}^{\sigma_{2}}$ and there exists at least one index $i$ such that $a_{2 i}\left(G_{1}^{\sigma_{1}}\right)<$ $a_{2 i}\left(G_{2}^{\sigma_{2}}\right)$, then we write that $G_{1}^{\sigma_{1}} \prec G_{2}^{\sigma_{2}}$. If $a_{2 i}\left(G_{1}^{\sigma_{1}}\right)=a_{2 i}\left(G_{2}^{\sigma_{2}}\right)$ for all $i$, we write that $G_{1}^{\sigma_{1}} \sim G_{2}^{\sigma_{2}}$. Note that there are non-isomorphic oriented graphs $G_{1}^{\sigma_{1}}$ and $G_{2}^{\sigma_{2}}$ with $G_{1}^{\sigma_{1}} \sim G_{2}^{\sigma_{2}}$, which implies that ' $\preceq$ ' is not a partial order in general.

According to the integral formula (1), we have for two oriented graphs $G_{1}^{\sigma_{1}}$ and $G_{2}^{\sigma_{2}}$ of order $n$ that

$$
G_{1}^{\sigma_{1}} \preceq G_{2}^{\sigma_{2}} \Longrightarrow \mathcal{E}_{s}\left(G_{1}^{\sigma_{1}}\right) \leq \mathcal{E}_{s}\left(G_{2}^{\sigma_{2}}\right)
$$

and

$$
G_{1}^{\sigma_{1}} \prec G_{2}^{\sigma_{2}} \Longrightarrow \mathcal{E}_{s}\left(G_{1}^{\sigma_{1}}\right)<\mathcal{E}_{s}\left(G_{2}^{\sigma_{2}}\right)
$$

Proposition 2.1 also implies that to study the spectral properties, as well as the skew energy, of an oriented graph, we need only consider the orientations of those arcs lying on even cycles. Let $G$ be a connected unicyclic graph whose unique cycle is even. Denote by $G^{+}$and $G^{-}$the oriented graph with underlying graph $G$ and the unique oriented cycle is evenly oriented and oddly oriented, respectively. Combining with Proposition 2.1 and Lemma 2.2, we have the following.

Theorem 2.3 Let $G$ be a connected unicyclic graph whose unique cycle $C$ is even. Then

$$
G^{-} \succ G^{+}
$$

Proof Let $C_{l}$ be the unique even cycle of $G$ with length $l$. By Proposition 2.1, we have

$$
\begin{aligned}
& a_{2 i}\left(G^{+}\right)=m(G, i)-2 m\left(G-C_{l}, i-\frac{l}{2}\right) ; \\
& a_{2 i}\left(G^{-}\right)=m(G, i)+2 m\left(G-C_{l}, i-\frac{l}{2}\right) .
\end{aligned}
$$

Then $a_{2 i}\left(G^{+}\right) \leq a_{2 i}\left(G^{-}\right)$and $a_{l}\left(G^{+}\right)<a_{l}\left(G^{-}\right)$. Thus, the result holds.

For convenience, denote by $U(n, d)$ the set of all oriented unicyclic graphs on $n$ vertices with diameter $d$ and by $T(n, d)$ the set of all undirected or oriented trees on $n$ vertices with diameter $d$. From Theorem 2.3, we can narrow down the possibility of the graph with minimum skew energy among all oriented unicyclic graphs as follows.

Lemma 2.4 Let $G^{\sigma}$ be an oriented graph with minimum skew energy among all graphs of $U(n, d)$. Denote by $C$ the unique oriented cycle of $G^{\sigma}$. Then $C$ is either an odd cycle or an evenly oriented even cycle.

Moreover, the following recursions concerning skew characteristic polynomials of oriented graphs are needed.

Lemma 2.5 Let $G^{\sigma}$ be an oriented unicyclic graph with skew characteristic polynomial

$$
\phi\left(G^{\sigma}, \lambda\right)=\sum_{i=0}^{n}(-1)^{i} a_{i} \lambda^{n-i}
$$


$C$ be the unique cycle of $G^{\sigma}$ and $e=(u, v)$ be an arc of $G^{\sigma}$. Suppose that $|C|=l$. Then

(a) $a_{i}\left(G^{\sigma}\right)=a_{i}\left(G^{\sigma}-e\right)+a_{i-2}\left(G^{\sigma}-u-v\right)+2 a_{i-l}\left(G^{\sigma}-V(C)\right)$ if $C$ is oddly oriented and $e \in C$;

(b) $a_{i}\left(G^{\sigma}\right)=a_{i}\left(G^{\sigma}-e\right)+a_{i-2}\left(G^{\sigma}-u-v\right)-2 a_{i-l}\left(G^{\sigma}-V(C)\right)$ if $C$ is evenly oriented and $e \in C$;

(c) $a_{i}\left(G^{\sigma}\right)=a_{i}\left(G^{\sigma}-e\right)+a_{i-2}\left(G^{\sigma}-u-v\right)$ otherwise.

Proof (a) We divide all basic subgraphs of $G^{\sigma}$ having $i$ vertices into three parts: those that do not contain the arc $e$; those that contain $e$ as the elementary subgraph $K_{2}$, and those that contain the evenly oriented cycle $C$. Then the former is the coefficient of $\lambda^{n-i}$ in $\phi\left(G^{\sigma}-e, \lambda\right)$, the second part can be considered as the coefficient of $\lambda^{n-i-2}$ in $\phi\left(G^{\sigma}-u-v, \lambda\right)$ and the latter can be considered as the coefficient of $\lambda^{n-i-l}$ in $\phi\left(G^{\sigma}-V(C), \lambda\right)$ multiplied by 2 . Hence, the equality holds.

Similarly, (b) and (c) can be proved.

Combining with Lemma 2.5(c) and (2), we have

Lemma 2.6 Let $G^{\sigma}$ be an oriented graph containing no even cycles and $G_{1}^{\sigma}$ a spanning subgraph (resp. proper spanning subgraph) of $G^{\sigma}$. Then $G^{\sigma} \succeq G_{1}^{\sigma}\left(\operatorname{resp} . G^{\sigma} \succ G_{1}^{\sigma}\right)$.

\section{The graphs with minimum skew energy among all oriented unicyclic graphs}

Denote by $T_{n, d}$ the tree obtained from the path $P_{d-1}$ and the star $S_{n-d+2}$ by identifying one pendent vertex of them, and by $U_{n, d}$ the undirected unicyclic graph obtained from the cycle $C_{4}$ by attaching a pendent vertex of the path $P_{d-2}$ and $n-d-1$ pendent edges to its two non-adjacent vertices, respectively; see Figure 1 . Note that both $T_{n, d}$ and $U_{n, d}$ contain $n$ vertices and have diameter $d$.

Let $v$ be an arbitrary vertex of the oriented graph $G^{\sigma}$. The operation by reversing the orientations of all arcs incident with $v$ and preserving the orientations of all its other arcs is called a reversal of $G^{\sigma}$ at $v$, the resultant graph is denoted by $G_{v}^{\sigma}$. Let $S\left(G^{\sigma}\right)$ and $S\left(G_{v}^{\sigma}\right)$ be the skew adjacency matrices of the oriented graphs $G^{\sigma}$ and $G_{v}^{\sigma}$, respectively. One can find that $S\left(G^{\sigma}\right)$ is similar to $S\left(G_{v}^{\sigma}\right)$, thus $G_{v}^{\sigma}$ has the same skew energy as that of $G^{\sigma}$. Especially, Adiga et al. [11] showed that the skew energy of a directed tree is independent of its orientation, which is equal to the energy of its underlying tree. Hence, the following results for undirected trees apply equally well to oriented trees, which will be cited in the following discussion directly.

Lemma 3.1 [18] For $2 \leq i \leq\left\lfloor\frac{n}{2}\right\rfloor$ and $n \geq 4$,

$$
P_{n} \succ P_{i} \cup P_{n-i} \succ P_{1} \cup P_{n-1} .
$$

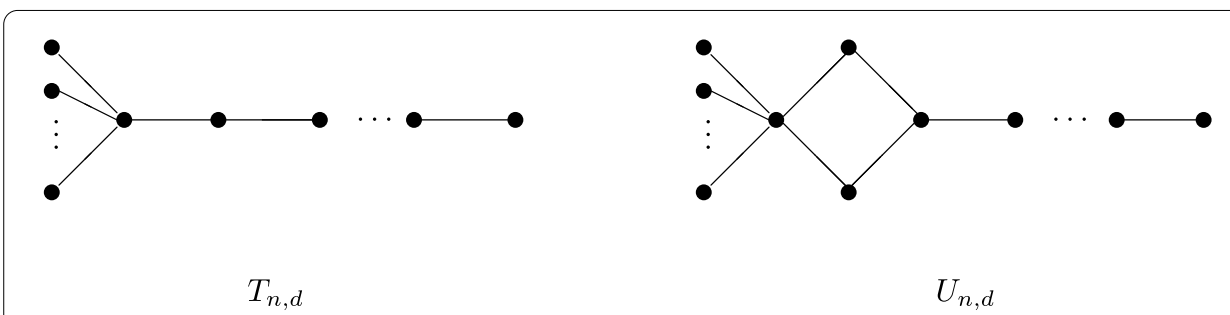

Figure 1 The tree $T_{n, d}$ and the unicyclic graph $U_{n, d}$. 
Lemma 3.2 [19] Let $n \geq 5, T_{n}$ denote any tree with order $n$ and $T_{n} \neq P_{n}, S_{n}$. Then

$$
P_{n} \succ T_{n} \succ S_{n}
$$

Lemma 3.3 [20] Let $T \in T(n, d)$ and $T \neq T_{n, d}$. Then

$$
T \succ T_{n, d} .
$$

Lemma 3.4 [21] If $d>d_{0} \geq 3$, then

$$
T_{n, d} \succ T_{n, d_{0}} .
$$

Denote by $U_{n, d}^{+}$the set of oriented graphs with underlying graph $U_{n, d}$ and the unique oriented cycle $C_{4}$ is evenly oriented. Because all skew adjacency matrices of $U_{n, d}^{+}$are similar by the method reversal above, and thus each of them has the same skew energy, we in the following do not concern the orientation of each graph of $U_{n, d}^{+}$. To obtain the main result of this paper, we first establish the following two lemmas.

Lemma 3.5 Let $n \geq 5$ and $G^{\sigma} \in U(n, n-2)$. If $G^{\sigma} \neq U_{n, n-2}^{+}$, then

$$
G^{\sigma} \succ U_{n, n-2}^{+}
$$

Proof By Lemma 2.4, it suffices to consider those oriented graphs whose unique cycle is either odd or evenly oriented.

We prove the result by induction on $n$. If $G^{\sigma} \in U(5,3)$ and $G^{\sigma} \neq U_{5,3}^{+}$, then $G^{\sigma}$ is isomorphic to either $G_{1}^{\sigma}$ or $G_{2}^{\sigma}$; if $G^{\sigma} \in U(6,4)$ and $G^{\sigma} \neq U_{6,4}^{+}$, then $G^{\sigma}$ is isomorphic to either $G_{3}^{\sigma}$ or $G_{4}^{\sigma}$ or $G_{5}^{\sigma}$; see Figure 2. (Here and in the sequel we do not consider the oriented graphs with oddly oriented cycle.) By a directly calculation, we have that

$$
\begin{aligned}
& \phi\left(G_{1}^{\sigma}, \lambda\right)=\lambda^{5}+5 \lambda^{3}+4 \lambda, \quad \phi\left(G_{2}^{\sigma}, \lambda\right)=\lambda^{5}+5 \lambda^{3}+3 \lambda, \\
& \phi\left(G_{3}^{\sigma}, \lambda\right)=\lambda^{6}+6 \lambda^{4}+8 \lambda^{2}+\lambda, \quad \phi\left(G_{4}^{\sigma}, \lambda\right)=\lambda^{6}+6 \lambda^{4}+7 \lambda^{2}+\lambda, \\
& \phi\left(G_{5}^{\sigma}, \lambda\right)=\lambda^{6}+6 \lambda^{4}+6 \lambda^{2}
\end{aligned}
$$

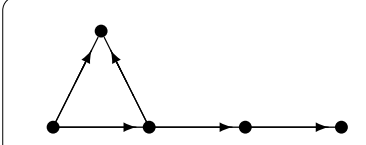

$G_{1}^{\sigma}$

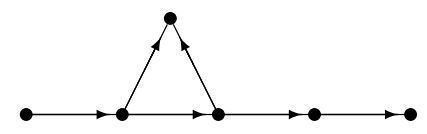

$G_{4}^{\sigma}$

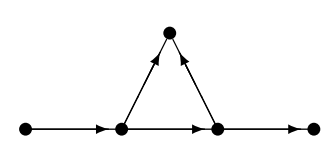

$G_{2}^{\sigma}$

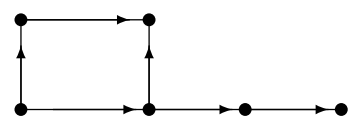

$G_{5}^{\sigma}$

Figure 2 Some oriented graphs for Lemma 3.5. 
and

$$
\phi\left(U_{5,3}^{+}, \lambda\right)=\lambda^{5}+5 \lambda^{3}+2 \lambda, \quad \phi\left(U_{6,4}^{+}, \lambda\right)=\lambda^{6}+6 \lambda^{4}+5 \lambda^{2} .
$$

Then by (2) the result follows for $n=5$ and 6.

Suppose that the result holds for graphs of $U(n-1, n-3)$ and $U(n-2, n-4)$ with $n \geq 7$. For $G^{\sigma} \in U(n, n-2)$, there exists pendent $\operatorname{arc} e=(u, v)$ such that $v$ is the pendent vertex of $G^{\sigma}$ and $u$ has degree 2 , since $d\left(G^{\sigma}\right)=n-2$, thus, the length of its unique cycle is not more than 4. Then $G^{\sigma}-v \in U(n-1, n-3)$ and $G^{\sigma}-v-u \in U(n-2, n-4)$. By Lemma 2.5(c), we have

$$
\begin{aligned}
& a_{i}\left(U_{n, n-2}^{+}\right)=a_{i}\left(U_{n-1, n-3}^{+}\right)+a_{i-2}\left(U_{n-2, n-4}^{+}\right), \\
& a_{i}\left(G^{\sigma}\right)=a_{i}\left(G^{\sigma}-v\right)+a_{i-2}\left(G^{\sigma}-v-u\right) .
\end{aligned}
$$

Combining with the induction hypothesis, $G^{\sigma}-v \succeq U_{n-1, n-3}^{+}$and $G^{\sigma}-v-u \succeq U_{n-2, n-4}^{+}$ with equality if and only if $G^{\sigma}-v=U_{n-1, n-3}^{+}$and $G^{\sigma}-v-u=U_{n-2, n-4}^{+}$, respectively. The proof is complete.

Lemma 3.6 Let $n \geq 6$ and $G^{\sigma} \in U(n, d)$ with $3 \leq d \leq n-3$. If $G^{\sigma} \neq U_{n, d}^{+}$, then

$$
G^{\sigma} \succ U_{n, d}^{+}
$$

Proof Similarly to Lemma 3.5, it suffices to consider those oriented graphs whose unique cycle is either odd or evenly oriented. Let $n-d=p$. We prove the lemma by induction on $p$. By Lemma 3.5, the result follows for $p=2$. Suppose now that $p \geq 3$ and the result holds for $n-d<p$.

Because $n-d=p \geq 3$, there is exactly one vertex in $U_{n, d}^{+}$with degree 3 . Let $u$ be such a vertex and $v$, adjacent to $u$, lie on the cycle $C_{4}$. Applying Lemma 2.5(b) to the arc $(u, v)$, we have

$$
a_{i}\left(U_{n, d}^{+}\right)=a_{i}\left(T_{n, d}\right)+a_{i-2}\left(P_{d-3} \cup S_{n-d+1}\right)-2 a_{i-4}\left(P_{d-3}\right) .
$$

Applying the recursion of Lemma 2.5(c) to $T_{n, d}$ and $P_{d-3} \cup S_{n-d+1}$ respectively, we have

$$
\begin{aligned}
\begin{array}{l}
a_{i}\left(T_{n, d}\right)=a_{i}\left(T_{n-1, d}\right) \\
=a_{i-2}\left(T_{n-1, d}\right)
\end{array} & +a_{i-2}\left(P_{d-2}\right)+a_{i-4}\left(P_{d-3}\right) ; \\
a_{i-2}\left(P_{d-3} \cup S_{n-d+1}\right) & =a_{i-2}\left(P_{d-3} \cup S_{n-d}\right)+a_{i-4}\left(P_{d-3} \cup(n-d-1) K_{1}\right) \\
= & a_{i-2}\left(P_{d-3} \cup S_{n-d}\right)+a_{i-4}\left(P_{d-3}\right) .
\end{aligned}
$$

Then another recursion for $a_{i}\left(U_{n, d}^{+}\right)$can be obtained as follows

$$
a_{i}\left(U_{n, d}^{+}\right)=a_{i}\left(T_{n-1, d}\right)+a_{i-2}\left(P_{d-2}\right)+a_{i-2}\left(P_{d-3} \cup S_{n-d}\right)
$$

Let $G^{\sigma} \in U(n, d)(3 \leq d \leq n-3, n \geq 6)$ and $G^{\sigma} \neq U_{n, d}^{+}$. To show $G^{\sigma} \succ U_{n, d}^{+}$, we divide $G^{\sigma}$ into the following three cases. 
Case $1 . G^{\sigma}$ contains no pendent vertices.

Then $G^{\sigma}=C_{n}$. Let $e=(u, v)$ be any arc of $C_{n}$. By Lemma 2.5,

$$
a_{i}\left(C_{n}\right)=a_{i}\left(C_{n}-e\right)+a_{i-2}\left(C_{n}-u-v\right)-2=a_{i}\left(P_{n}\right)+a_{i-2}\left(P_{n-2}\right)-2,
$$

if $i=n$ and $C_{n}$ is evenly oriented; otherwise

$$
a_{i}\left(C_{n}\right)=a_{i}\left(C_{n}-e\right)+a_{i-2}\left(C_{n}-u-v\right)=a_{i}\left(P_{n}\right)+a_{i-2}\left(P_{n-2}\right) .
$$

By Lemma 3.2, $a_{i}\left(P_{n}\right)>a_{i}\left(T_{n, d}\right)$ and $a_{i-4}\left(P_{d-3}\right) \geq 1$. By Lemma 3.1, $a_{i-2}\left(P_{n-2}\right) \geq a_{i-2}\left(P_{d-3} \cup\right.$ $\left.S_{n-d+1}\right)$. Then combining with (3), (5) and (6), $G^{\sigma} \succ U_{n, d}^{+}$.

Case 2. All pendent vertices are contained in the longest path of $G^{\sigma}$.

Let, in $G^{\sigma}, C_{r}(r<n)$ be the unique cycle and $P\left(G^{\sigma}\right)=v_{0} v_{1} \cdots v_{k}$ be the longest path. Then $k=d$, and either $v_{0}$ or $v_{d}$ is the pendent vertex of $G^{\sigma}$. Recall that $p \geq 3$, then there exist two adjacent vertices, say $u$ and $v$, are not contained in $P\left(G^{\sigma}\right)$. Hence, both $u$ and $v$, are contained in $C_{r}$. Consequently, $G^{\sigma}-(u, v) \in T\left(n, d_{1}\right), G^{\sigma}-v \in T\left(n-1, d_{2}\right)$ and $G^{\sigma}-v-u \in$ $T\left(n-2, d_{3}\right)$. Since $P\left(G^{\sigma}\right)$ still is contained in $G^{\sigma}-(u, v), G^{\sigma}-v$ and $G^{\sigma}-v-u$, we have $d_{i} \geq d$ for $i=1,2,3$. Then by Lemmas 3.3 and 3.4 ,

$$
G^{\sigma}-(u, v) \succeq T_{n, d_{1}} \succeq T_{n, d}, \quad G^{\sigma}-v \succeq T_{n-1, d_{2}} \succeq T_{n-1, d}
$$

and

$$
G^{\sigma}-v-u \succeq T_{n-2, d_{3}} \succeq T_{n-2, d} \succ P_{d-3} \cup P_{n-d+1} \succ P_{d-3} \cup S_{n-d+1}
$$

Subcase 2.1. $r$ is odd.

Combining with (3), (7), (8) and Lemma 2.5(c), we have

$$
\begin{aligned}
a_{i}\left(G^{\sigma}\right) & =a_{i}\left(G^{\sigma}-(u, v)\right)+a_{i-2}\left(G^{\sigma}-u-v\right) \\
& >a_{i}\left(T_{n, d}\right)+a_{i-2}\left(P_{d-3} \cup S_{n-d+1}\right)>a_{i}\left(U_{n, d}^{+}\right) .
\end{aligned}
$$

Hence, $G^{\sigma} \succ U_{n, d}^{+}$.

Subcase 2.2. $r$ is even and $r>4$.

Then there exist three vertices, say $u, v$ and $w$, such that each of them is not contained in $P\left(G^{\sigma}\right)$ and $v$ adjacent to both $u$ and $w$. Further, let $u_{1}(\neq v)$ adjacent to $u$ and $w_{1}(\neq v)$ adjacent to $w$. Then $G^{\sigma}-v-u-w-w_{1} \succ G^{\sigma}-C_{r}, G^{\sigma}-v-u-w-u_{1} \succ G^{\sigma}-C_{r}$ and $G^{\sigma}-w-u-v \in T\left(n-3, d_{4}\right)$ with $d_{4} \geq d$. By Lemma 2.5(b), we have

$$
a_{i}\left(G^{\sigma}\right)=a_{i}\left(G^{\sigma}-(u, v)\right)+a_{i-2}\left(G^{\sigma}-u-v\right)-2 a_{i-r}\left(G^{\sigma}-C_{r}\right) .
$$

Applying Lemma 2.5(c) repeatedly, we have

$$
\begin{aligned}
a_{i}\left(G^{\sigma}-(u, v)\right) & =a_{i}\left(G^{\sigma}-v\right)+a_{i-2}\left(G^{\sigma}-v-w\right) \\
& =a_{i}\left(G^{\sigma}-v\right)+a_{i-2}\left(G^{\sigma}-u-v-w\right)+a_{i-4}\left(G^{\sigma}-u_{1}-u-v-w\right) \\
& \geq a_{i}\left(G^{\sigma}-v\right)+a_{i-2}\left(G^{\sigma}-u-v-w\right)+a_{i-r}\left(G^{\sigma}-C_{r}\right),
\end{aligned}
$$




$$
\begin{aligned}
a_{i-2}\left(G^{\sigma}-u-v\right) & =a_{i-2}\left(G^{\sigma}-w-u-v\right)+a_{i-4}\left(G^{\sigma}-w_{1}-w-u-v\right) \\
& \geq a_{i-2}\left(G^{\sigma}-w-u-v\right)+a_{i-r}\left(G^{\sigma}-C_{r}\right) .
\end{aligned}
$$

Then combining with (7), (9) and Lemma 3.4

$$
a_{i}\left(G^{\sigma}\right) \geq a_{i}\left(G^{\sigma}-v\right)+2 a_{i-2}\left(G^{\sigma}-u-v-w\right) \geq a_{i}\left(T_{n-1, d}\right)+2 a_{i-2}\left(T_{n-3, d}\right) .
$$

From Lemma 2.6, we have $a_{i-2}\left(T_{n-3, d}\right)>a_{i-2}\left(P_{d-3} \cup S_{n-d}\right)$ and $a_{i-2}\left(T_{n-3, d}\right)>a_{i-2}\left(P_{d-2}\right)$. Consequently, combining with (4), $G^{\sigma} \succ U_{n, d}^{+}$.

Subcase 2.3. $r=4$.

Recall that $p \geq 3$, then $C_{r}$ adjoint to $P\left(G^{\sigma}\right)$ at most one arc. If $C_{r}$ and $P\left(G^{\sigma}\right)$ have no common arc, then there exist three vertices, say $u, v$ and $w$, such that each of them is not contained in $P\left(G^{\sigma}\right)$ and $v$ adjacent to both $u$ and $w$. Then $G^{\sigma} \succ U_{n, d}^{+}$by the discussion similar to Subcase 2.2. If $C_{r}$ and $P\left(G^{\sigma}\right)$ have exactly one common arc, then $d=n-3$. Let $e=(u, v)$ be the unique arc of $C_{4}$ which has no common vertices with $P\left(G^{\sigma}\right)$. Applying Lemmas $2.5,3.1,3.3$ and (4), we have for some $j$ with $1 \leq j \leq d-1$

$$
\begin{aligned}
a_{i}\left(G^{\sigma}\right)= & a_{i}\left(G^{\sigma}-(u, v)\right)+a_{i-2}\left(G^{\sigma}-u-v\right)-2 a_{i-4}\left(G^{\sigma}-C_{4}\right) \\
= & a_{i}\left(G^{\sigma}-v\right)+a_{i-2}\left(P_{j} \cup P_{n-2-j}\right)+a_{i-2}\left(P_{n-2}\right)-2 a_{i-4}\left(P_{j} \cup P_{n-4-j}\right) \\
= & a_{i}\left(G^{\sigma}-v\right)+a_{i-2}\left(P_{j} \cup P_{n-3-j}\right)+a_{i-4}\left(P_{j} \cup P_{n-4-j}\right) \\
& +a_{i-2}\left(P_{n-3}\right)+a_{i-4}\left(P_{n-4}\right)-2 a_{i-4}\left(P_{j} \cup P_{n-4-j}\right) \\
= & a_{i}\left(G^{\sigma}-v\right)+a_{i-2}\left(P_{j} \cup P_{n-3-j}\right)+a_{i-2}\left(P_{n-3}\right)+a_{i-4}\left(P_{n-4}\right)-a_{i-4}\left(P_{j} \cup P_{n-4-j}\right) \\
> & a_{i}\left(G^{\sigma}-v\right)+a_{i-2}\left(P_{j} \cup P_{n-3-j}\right)+a_{i-2}\left(P_{n-3}\right) \\
> & a_{i}\left(U_{n-1, n-3}^{+}\right)+a_{i-2}\left(P_{n-4}\right)+a_{i-2}\left(P_{n-3}\right) .
\end{aligned}
$$

On the other hand, we have

$$
\begin{aligned}
a_{i}\left(U_{n, n-3}^{+}\right) & =a_{i}\left(U_{n-1, n-3}^{+}\right)+a_{i-2}\left(P_{1} \cup T_{n-3, n-5}\right) \leq a_{i}\left(U_{n-1, n-3}^{+}\right)+2 a_{i-2}\left(P_{n-4}\right) \\
& <a_{i}\left(U_{n-1, n-3}^{+}\right)+a_{i-2}\left(P_{n-4}\right)+a_{i-2}\left(P_{n-3}\right) .
\end{aligned}
$$

Consequently, $G^{\sigma} \succ U_{n, d}^{+}$.

Case 3. There exist pendent vertices not contained in the longest path of $G^{\sigma}$.

Let $P\left(G^{\sigma}\right)=v_{0} v_{1} \cdots v_{k}$ be the longest path of $G^{\sigma}$, which is defined as Case 2, and $u \notin P\left(G^{\sigma}\right)$, a pendent vertex of $G^{\sigma}$. Suppose that $v$ is the unique neighbor of $u$. By Lemma 2.5(c),

$$
a_{i}\left(G^{\sigma}\right)=a_{i}\left(G^{\sigma}-u\right)+a_{i-2}\left(G^{\sigma}-u-v\right)
$$

Let $v_{1}$ be the vertex of $U_{n, d}^{+}$with degree $n-d-1$ and $u_{1}$, adjacent to $v_{1}$, the pendent vertex. Then

$$
\begin{aligned}
a_{i}\left(U_{n, d}^{+}\right) & =a_{i}\left(U_{n, d}^{+}-u_{1}\right)+a_{i-2}\left(U_{n, d}^{+}-v_{1}-u_{1}\right) \\
& =a_{i}\left(U_{n-1, d}^{+}\right)+a_{i-2}\left((n-d-2) P_{1} \cup T_{d, d-2}\right) .
\end{aligned}
$$


Subcase 3.1. There exists a pendent vertex such that its neighbor is not contained in $P\left(G^{\sigma}\right)$

Say $u$ is such a pendent vertex. Then $G^{\sigma}-u \in U(n-1, d)$, and thus $a_{i}\left(G^{\sigma}-u\right) \geq a_{i}\left(U_{n-1, d}^{+}\right)$ by the induction hypothesis; $G^{\sigma}-u-v \succeq(n-d-3) P_{1} \cup P_{d+1} \succ(n-d-2) P_{1} \cup T_{d, d-2}$ by Lemmas 3.3 and 2.6. Consequently, $G^{\sigma} \succ U_{n, d}^{+}$.

Subcase 3.2. The neighbor of each pendent vertex is contained in $P\left(G^{\sigma}\right)$, and there exist no pendent vertices whose neighbor lies on $C_{r}$.

Without loss of generality, let $j(1 \leq j \leq d-1)$ be the least index such that each vertex of $\left\{v_{1}, v_{2}, \ldots, v_{j-1}\right\}$ has degree 2 and $v$, a pendent vertex, adjacent to $v_{j}$. Then $G^{\sigma}-u-v_{j} \supseteq$ $P_{j} \cup G_{1}^{\sigma}$, where $G_{1}^{\sigma} \in U\left(s, d^{*}\right)$ with $j+s \leq n-2$ and $d^{*} \geq d-j-1$. Then by Lemma 3.3 and the induction hypothesis, we have $G_{1}^{\sigma} \succeq U_{d-j+1, d-j-1}^{+}$. Moreover,

$$
\begin{aligned}
a_{i}\left(P_{j} \cup U_{d-j+1, d-j-1}^{+}\right)= & a_{i}\left(P_{j} \cup T_{d-j+1, d-j-1}\right)+a_{i-2}\left(P_{j} \cup P_{3} \cup P_{d-j-4}\right)-2 a_{i-4}\left(P_{j} \cup P_{d-j-4}\right) \\
= & a_{i}\left(P_{j} \cup P_{d-j}\right)+a_{i-2}\left(P_{j} \cup P_{d-j-2}\right)+a_{i-2}\left(P_{j} \cup P_{2} \cup P_{d-j-4}\right) \\
& +a_{i-4}\left(P_{j} \cup P_{1} \cup P_{d-j-4}\right)-2 a_{i-4}\left(P_{j} \cup P_{d-j-4}\right) \\
= & a_{i}\left(P_{j} \cup P_{d-j}\right)+a_{i-2}\left(P_{j} \cup P_{d-j-2}\right)+a_{i-2}\left(P_{j} \cup P_{d-j-4}\right) \\
\geq & a_{i}\left(P_{j} \cup P_{d-j}\right)+a_{i-2}\left(P_{j} \cup P_{d-j-2}\right) \\
> & a_{i}\left(P_{d-1}\right)+a_{i-2}\left(P_{d-3}\right) \quad(\text { By Lemma 3.1) } \\
= & a_{i}\left(T_{d, d-2}\right) .
\end{aligned}
$$

Thus, $G^{\sigma}-u-v \succ T_{d, d-2}$. Consequently, $G^{\sigma} \succ U_{n, d}^{+}$by (11).

Subcase 3.3. The neighbor of each pendent vertex is contained in $P\left(G^{\sigma}\right)$, and there exist pendent vertices whose neighbor lies on $C_{r}$.

Let now $v_{l}, v_{l+1}, \ldots, v_{l+s}(s \geq 0)$ be all common vertices of $P\left(G^{\sigma}\right)$ and $C_{r}$. If $s=0$, then $v=v_{l}$, i.e., $v$ is the unique common vertex of $P\left(G^{\sigma}\right)$ and $C_{r}$. Then $G^{\sigma}-u-v \supseteq P_{l} \cup P_{d-l} \cup P_{r-1}$ and thus

$$
\begin{aligned}
a_{i}\left(G^{\sigma}-u-v\right) & \geq a_{i}\left(P_{l} \cup P_{d-l} \cup P_{r-1}\right) \\
& =a_{i}\left(P_{l} \cup P_{d-l} \cup P_{r-2}\right)+a_{i-2}\left(P_{l} \cup P_{d-l} \cup P_{r-2}\right) \\
& >a_{i}\left(P_{d-1}\right)+a_{i-2}\left(P_{d-3}\right) \\
& =a_{i}\left(T_{d, d-2}\right) .
\end{aligned}
$$

Consequently, $G^{\sigma} \succ U_{n, d}^{+}$. If $s>0$ and $v \neq v_{l}, v_{l+s}$, then $G^{\sigma}-u-v \supseteq P_{d+l}$, and thus $G^{\sigma}-$ $u-v>T_{d, d-2}$. Hence, $G^{\sigma}>U_{n, d}^{+}$. If $s>0$, and $v=v_{l}$ or $v=v_{l+s}$. (Without loss of generality, suppose that $v=v_{l}$.) Then $G^{\sigma}-u-v \supseteq P_{l} \cup T_{1}$, where $T_{1}$ denotes the tree obtained from the path $v_{l+1} \cdots v_{l+s}$ by adding a pendent vertex. Similarly, we have

$$
\begin{aligned}
a_{i}\left(P_{l} \cup T_{1}\right) & =a_{i}\left(P_{l} \cup P_{d-l}\right)+a_{i-2}\left(P_{l} \cup P_{s-1} \cup P_{d-l-s}\right) \\
& >a_{i}\left(P_{d-1}\right)+a_{i-2}\left(P_{d-3}\right) \\
& =a_{i}\left(T_{d, d-2}\right) .
\end{aligned}
$$

Hence, $G^{\sigma} \succ U_{n, d}^{+}$.

Thus the result follows. 
Putting Lemma 3.5 together with Lemma 3.6, the main result of this paper can be obtained.

Theorem 3.7 Let $n \geq 6$ and $3 \leq d \leq n-2$. Then the oriented graph with minimum skew energy among all graphs of $U(n, d)$ is of $U_{n, d}^{+}$.

Proof Let $n \geq 6$ and $3 \leq d \leq n-2$. Then $G^{\sigma} \geq U_{n, d}^{+}$with equality if and only if $G^{\sigma}=U_{n, d}^{+}$for any oriented unicyclic graph $G^{\sigma} \in U(n, d)$ by Lemma 3.5 and Lemma 3.6. Then $\mathcal{E}_{s}\left(G^{\sigma}\right) \geq$ $\mathcal{E}_{s}\left(U_{n, d}^{+}\right)$with equality if and only if $G^{\sigma}=U_{n, d}^{+}$by $(2)$, and thus the result follows.

\section{Competing interests}

The authors declare that they have no competing interests.

\section{Authors' contributions}

S-CG wrote and reformed the article. All authors read and approved the final manuscript.

\section{Acknowledgements}

This work was supported by the National Natural Science Foundation of China (11171373) and the Zhejiang Provincial Natural Science Foundation of China (LY12A01016).

Received: 1 May 2013 Accepted: 16 August 2013 Published: 2 September 2013

\section{References}

1. Gutman, I: The energy of a graph. Ber. Math.-Stat. Sekt. Forsch. Graz 103, 1-22 (1978)

2. Coulson, CA: On the calculation of the energy in unsaturated hydrocarbon molecules. Proc. Camb. Philos. Soc. 36, 201-203 (1940)

3. Akbari, S, Ghorbani, E, Oboudi, MR: Edge addition, singular values and energy of graphs and matrices. Linear Algebra Appl. 430, 2192-2199 (2009)

4. Blackburn, SR, Shparlinski, IE: On the average energy of circulant graphs. Linear Algebra Appl. 428, 1956-1963 (2008)

5. Gutman, I: The energy of a graph: old and new results. In: Better, A, Kohnert, A, Lau, R, Wassermann, A (eds.) Algebraic Combinatorics and Applications, pp. 196-211. Springer, Berlin (2001)

6. Indulal, G, Vijayakumar, A: A note on energy of some graphs. MATCH Commun. Math. Comput. Chem. 59, 269-274 (2008)

7. Majstorović, S, Klobučar, A, Gutman, I: Selected topics from the theory of graph energy: hypoenergetic graphs. In: Cvetković, D, Gutman, I (eds.) Applications of Graph Spectra, pp. 65-105. Math. Inst., Belgrade (2009)

8. Nikiforov, V: The energy of graphs and matrices. J. Math. Anal. Appl. 326, 1472-1475 (2007)

9. Gutman, I, Zhou, B: Laplacian energy of a graph. Linear Algebra Appl. 414, 29-37 (2006)

10. Gutman, I, Robbiano, M, Martins, EA, Cardoso, DM, Medina, L, Rojo, O: Energy of line graphs. Linear Algebra Appl. 433, 1312-1323 (2010)

11. Adiga, C, Balakrishnan, R, So, W: The skew energy of a digraph. Linear Algebra Appl. 432, 1825-1835 (2010)

12. Hou, YP, Shen, $X L$, Zhang, $C Y$ : Oriented unicyclic graphs with extremal skew energy. arXiv: 1108.6229

13. Gong, SC, Li, XL, Xu, GH: On oriented graphs with minimal skew energy. arXiv:1304.2458

14. Gong, SC, Li, XL, Xu, GH: 3-Regular oriented graphs with optimum skew energy. Linear Algebra Appl. 436, 465-471 (2012)

15. Cvetković, D, Doob, M, Sachs, H: Spectra of Graphs. Academic Press, New York (1980)

16. Gong, SC, Xu, GH: The characteristic polynomial and the matchings polynomial of a weighted oriented graph. Linear Algebra Appl. 436, 3597-3607 (2012)

17. Hou, Y, Lei, T: Characteristic polynomials of skew-adjacency matrices of oriented graphs. Electron. J. Comb. 18, 156 (2011)

18. Zhou, B, Li, F: On minimal energies of trees of a prescribed diameter. J. Math. Chem. 39, 465-473 (2006)

19. Gutman, I: Acyclic systems with extremal Hückel-electron energy. Theor. Chim. Acta 45, $79-87$ (1977)

20. Yan, W, Ye, L: On the minimal energy of trees with a given diameter. Appl. Math. Lett. 18, 1046-1052 (2005)

21. Li, F, Zhou, B: Minimal energy of unicyclic graphs of a given diameter. J. Math. Chem. 39, 465-473 (2006) 